\title{
Apoptose na infecção experimental de cães domésticos com Ehrlichia canis
}

\author{
Apoptosis in experimental infection with Ehrlichia canis in domestic dogs
}

\author{
Irma Ximena Barbosa Sanchez ${ }^{\mathrm{I}, \mathrm{III}}$ Teresa de Jesus Oviedo Socarras ${ }^{\mathrm{II}, \mathrm{III}}$ Miguel Ladino Silva ${ }^{\mathrm{IV}}$ \\ Nubia Braga Pereira ${ }^{\mathrm{III}}$ Rosangela Zacarias Machado ${ }^{\mathrm{V}}$ Anilton Cesar Vasconcelos ${ }^{\mathrm{III}}$
}

\section{RESUMO}

A Erliquiose canina é uma zoonose causada pela Ehrlichia canis, bactéria Gram negativa de distribuição mundial. Alguns cães com erliquiose se tornam portadores assintomáticos enquanto outros desenvolvem uma doença aguda com morte rápida. A apoptose pode ser importante na eliminação de patógenos intracelulares, podendo, nas infecções por Ehrlichia sp. e Anaplasma sp., ocorrer modulação da apoptose celular para prolongar a sobrevivência desses organismos. Para avaliação do papel da apoptose na erliquiose canina, sete cães foram distribuídos em dois grupos. No Grupo inoculado, realizou-se a infecção por via intravenosa com sangue infectado com E. canis (isolado Jaboticabal), sendo realizada a inoculação com $P B S$ estéril nos animais pertencentes ao Grupo Controle. Semanalmente e até 35 dias pós-inoculação, amostras de sangue foram coletadas e submetidas a n-PCR e reação de imunofluorescência (RIFI) para confirmação da infecção. No $36^{\circ}$ dia pós-inoculação, os animais foram eutanasiados, sendo as amostras de baço, linfonodo, rim e figado coletadas e processadas para as técnicas de TUNEL e imunohistoquímica (Bcl-2, Bax). Verificou-se pela $n-P C R$ que os animais inoculados se tornaram positivos para E. canis a partir da segunda semana. Pela RIFI, verificou-se animais com sorologia positiva a partir da terceira semana pós-inoculação. No grupo controle, todos os testes realizados foram negativos para E. canis. Apesar da reação de TUNEL mostrar maior incidência de apoptose no Grupo Inoculado, sendo o baço e os linfonodos os órgãos que apresentaram maior marcação, os resultados da imunohistoquímica para Bcl-2 e Bax indicam que a via intrínseca de apoptose não é importante nos órgãos analisados.
Palavras-chave: apoptose, Ehrlichia canis, n-PCR, RIFI, TUNEL, Bcl-2, Bax.

\section{ABSTRACT}

Some dogs infected with Ehrlichia canis become asymptomatic while others develop an acute illness followed by quick death. Apoptosis may be an important mechanism for elimination of intracellular pathogens. Also, Ehrlichia sp. and Anaplasma sp. can modulate apoptosis to extend their survival. To evaluate the role of apoptosis in canine ehrlichiosis, 7 dogs were assigned into 2 groups, one with 4 animals inoculated intravenously with blood infected with Ehrlichia canis (Jaboticabal isolate) and a control with 3 dogs, inoculated with sterile PBS. Blood samples were collected weekly and 35 days post-inoculation to confirm the infection by $n P C R$ and immunofluorescence. Thirty-six days after inoculation the animals were euthanized and samples from spleen, lymph nodes, kidney and liver were collected to carry out the TUNEL technique and immunohistochemistry (Bcl-2, Bax). Inoculated animals became positive for $\boldsymbol{E}$. canis by $n P C R$ already in the second week and by immunofluorescence in the third week after inoculation. The control group showed negative for $\boldsymbol{E}$. canis in all tests. The TUNEL reaction showed a higher incidence of apoptosis in the inoculated group, with stronger labeling in the spleen and lymph nodes. The results of immunohistochemistry for Bcl-2 and Bax suggest that the intrinsic pathway of apoptosis is not important in the analyzed organs.

Key words: apoptosis, Ehrlichia canis, n-PCR, IFA, TUNEL, Bcl-2, Bax.

\footnotetext{
'Laboratorio de Patologías, Facultad de Medicina Veterinaria y Zootecnia (FMVZ), Universidad del Tolima (UT), Altos de Santa Helena, 7652000, Ibagué, Tolima, Colombia. E-mail: isanchez@ut.edu.co. Autor para correspondência.

"Departamento de Sanidad Animal, Facultad de Medicina Veterinaria y Zootecnia, Universidad de Córdoba (UCO), Montería, Colombia.

IIIDepartamento de Patologia Geral, Instituto de Ciências Biológicas (ICB), Universidade Federal de Minas Gerais (UFMG), Belo Horizonte, MG, Brasil.

${ }^{\text {IV }}$ Departamento de Clínica e Cirurgia Veterinária, Faculdade de Ciências Agrárias e Veterinárias (FCAV), Universidade Estadual Paulista (UNESP), Jaboticabal, SP, Brasil

vDepartamento de Patologia Veterinária, FCAV, UNESP, Jaboticabal, SP, Brasil.
} 


\section{INTRODUÇÃO}

A trombocitopenia tropical canina é causada por Ehrlichia canis, bactéria Gram negativa, intracitoplasmática obrigatória (MANNA et al., 2004; YU XUE-JIE et al., 2007) da ordem Rickettsiales e família Anaplasmataceae (DUMLER et al., 2001), sendo encontrada no citoplasma dos mononucleares dos cães infectados, com transmissão pela picada do carrapato Rhipicephalus sanguineus infectado (LABRUNA \& PEREIRA, 2001; MELO et al., 2011).

Essa bactéria apresenta genoma mundialmente bem conservado, visto o gene 16S rRNA ser 99,9 a 100\% idêntico nos diferentes isolados das Américas do Sul e do Norte e da Ásia (SIARKOU et al., 2007). AGUIAR et al. (2007), comparando o genótipo de uma cepa isolada no ano de 1993 na cidade de Jaboticabal, estado de São Paulo, Brasil, com a sequência Norte-Americana/Oklahoma disponível no GeneBank sob o registro M73221.1, (http:// www.ncbi.nlm.nih.gov/nuccore/M73221.1) considerada padrão mundial para o diagnóstico sorológico, encontraram $100 \%$ de similaridade entre elas.

A resposta imunológica não se encontra ainda bem caracterizada em cães infectados pela $\boldsymbol{E}$. canis (HESS et al., 2006). Cães infectados e assintomáticos provavelmente ocorrem devido à tolerância ou à inadequada defesa imunológica (RIKIHISA, 2006). Por outro lado, a apoptose tem importante papel na regulação da resposta imunológica e na eliminação e infecção de patógenos intracelulares (GRASSMÉ et al., 2001). A célula infectada pode sofrer apoptose na presença de vários agentes, embora alguns patógenos sejam capazes de inibi- la (DELEO, 2004). Estudos recentes têm demonstrado que os gêneros Ehrlichia e Anaplasma possuem sofisticados mecanismos de evasão do sistema imune inato. $\mathrm{Na}$ infecção por Anaplasma phagocytophilum, verificase inibição da apoptose espontânea de neutrófilos humanos através do bloqueio de Fas (CD95/Apo-1) (BORJESSON et al., 2005). Em comparação, na infecção por Ehrlichia chaffeensis, observa-se a capacidade de modulação da ação de inibidores da NF- $\kappa$ B e da apoptose (ZHANG et al., 2004), inibindo a apoptose nas celulas infectadas (RIKIHISA, 2006).

O presente trabalho avaliou e quantificou a apoptose nas células do baço, linfonodos, fígado e rim de cães inoculados experimentalmente com E. canis, utilizando a reação de TUNEL para a sua detecção. Além disso, utilizou-se da imunohistoquímica para as moléculas Bax e Bcl-2, pró e anti-apoptóticas, respectivamente, para se verificar o envolvimento da via intrínseca .

\section{MATERIAL E MÉTODOS}

Utilizou-se a amostra de $\boldsymbol{E}$. canis isolado Jaboticabal, obtida no ano de 1993 e mantida na forma de sangue congelado na Faculdade de Ciências Agrárias e Veterinárias (FCAV) da Universidade Estadual Paulista (UNESP), em Jaboticabal, estado de São Paulo (MACHADO, R.Z., dados não publicados).

\section{Animais experimentais}

Sete cães sem raça definida (SRD), provenientes e mantidos no Canil de Experimentação da UNESP, Campus Jaboticabal, clinicamente saudáveis e livres de carrapatos, com idades entre seis a oito meses, foram aleatoriamente alocados em dois grupos: um de quatro (G I - Inoculado) e outro de três animais (G II Controle). Cada cão no $\mathrm{G}$ I foi inoculado via endovenosa com $3 \mathrm{ml}$ de sangue infectado (cepa Jaboticabal), e em cada animal do G II foi injetado via intravenosa com $3 \mathrm{ml}$ de PBS estéril. Todos os animais foram acompanhados por 35 dias após a aplicação, mantidos em isolamento sob condições controladas de temperatura ambiente, sendo alimentados com ração comercial duas vezes ao dia e recebendo água ad libitum.

Do nascimento até o início do experimento, todos os animais foram mensalmente submetidos à coleta de fezes para o diagnóstico e tratamento de parasitas intestinais. Uma semana antes da inoculação experimental, os animais foram submetidos a coletas de sangue para testes sorológicos (Reação de Imunofluorescência Indireta - RIFI), para confirmação de ausência de infecção ou contato prévio com Babesia canis, Leishmania spp. e E. canis. Os animais e o canil receberam aplicação de carrapaticida mensal e quinzenalmente, respectivamente.

\section{Coleção de amostras sanguíneas}

Amostras do sangue venoso foram coletadas de todos os cães em tubos com e sem EDTA (Becton Dickinson, Meylan, França), mediante venopunção cefálica ou jugular no dia 0 (antes da inoculação) e a cada sete dias, até os 35 dias pós inoculação. Essas amostras foram utilizadas para a detecção de inclusões em células mononucleares do sangue periférico (peripheral blood mononuclear cell, PBMC) pela coloração de Rosenfeld e para a extração de DNA a ser submetido à n-PCR para diagnóstico de E. canis. Os soros obtidos foram utilizados para realização da RIFI para diagnóstico de $\boldsymbol{E}$. canis.

Amostras de tecidos

Trinta e seis dias após a inoculação, os animais de ambos os grupos foram eutanasiados por 
super-dosagem de tiopental sódico 7,5\% $\left(75 \mathrm{mg} \mathrm{kg}^{-1}\right)$, sendo coletadas e processadas amostras de baço, fígado, rim e linfonodos para a reação de TUNEL (Terminal deoxinucleotidil transferase Uracil Nick End Labeling) e de imunohistoquímica para a detecção da presença de Bax e Bcl-2. As imagens obtidas da técnica de TUNEL foram submetidas à morfometria para quantificação do índice apoptótico em analisador de imagens (Image Pro-Plus versão 4.5.0.29 para Windows $^{\circledR}$, Media Cybernetics Inc, EUA).

Avaliações de apoptose

Determinação do índice apoptótico em amostragem A quantificação da apoptose foi feita em amostras de baço, fígado, rim e linfonodos de animais dos grupos I e II, submetidas à reação de TUNEL, sendo o índice apoptótico obtido pela fórmula:

Índice apoptótico $(\mathrm{IA})=\left(\mathrm{n} \mathrm{n}^{-}\right.$de células TUNEL positivo/ $\sum \mathrm{n}^{\mathrm{o}}$ de células totais $)$ x 100

Marcação in situ para detecção de apoptose pela reação de TUNEL

Utilizou-se kit comercial para a detecção da fragmentação do genoma (TdT-FragEL TM $D N A$ Fragmentation Detection Kit, Calbiochem ${ }^{\circledR}$, EUA). Lâminas carregadas positivamente (CMS-2, Amitel ${ }^{\circledR}$, Brasil), contendo as secções das amostras dos órgãos coletados foram processadas de acordo com as instruções do fabricante. Quantificou-se o número de células mononucleares positivas à reação de TUNEL no número mínimo de campos achados para cada amostra, como descrito por MORO et al. (2003), sendo o resultado expresso em porcentagem (IA) para cada órgão.

Reação imunohistoquímica com o anticorpo anti-Bax Para a reação com o anticorpo anti-Bax, seguiu-se o protocolo utilizado por RODRIGUES et al. (2011). As lâminas foram incubadas em xilol, seguidas de lavagem em série decrescente de etanol $(100 \%, 90 \%$, $80 \%$ e $70 \%$ ) e PBS 1x. Para a recuperação antigênica, utilizou-se ácido cítrico $10 \mathrm{mM} \mathrm{pH} 6.0 \mathrm{em}$ banho úmido por 20 minutos a $95^{\circ} \mathrm{C}$. Para o bloqueio da peroxidase endógena, foi utilizado Peroxidase block (Peroxidase Detection System, Novocastra ${ }^{\mathrm{TM}}$, Inglaterra) por 15 minutos, em temperatura ambiente, sendo as lâminas enxaguadas em PBS e a seguir incubadas com anticorpo primário anti-Bax (Polyclonal Rabbit Anti-Human Bax, Dako $^{\circledR}$, Dinamarca) na diluição de 1:1.000, durante toda a noite em temperatura ambiente. A seguir, foram lavadas com PBS e incubadas com o anticorpo secundário biotinilado (Peroxidase Detection System, Novocastra $^{\mathrm{TM}}$, Inglaterra) por uma hora em temperatura ambiente. Posteriormente, as lâminas foram enxaguadas com PBS, seguida da aplicação de Streptoavidina-HRP (Peroxidase Detection System, Novocastra ${ }^{\mathrm{TM}}$, Inglaterra) em câmara úmida por 30 minutos, à temperatura ambiente. Para a revelação, utilizou-se $D A B$ Chromogen na diluição de 1:20 por 5 minutos. Como contra-coloração, foi utilizada hematoxilina de Mayer's por 15 segundos.

Reação imunohistoquímica com o anticorpo anti-Bcl-2 Empregou-se o mesmo protocolo, utilizandose como anticorpo primário anti-Bcl-2 (Monoclonal mouse anti-human BCl2 Oncoprotein clone 124, Dako $^{\circledR}$, Dinamarca) na diluição de 1:200. Além disso, a solução de recuperação antigênica foi trocada por Tris EDTA pH 8,0 (Tris HCL 0,152g, Tris Base 1,094g, EDTA $3,36 \mathrm{~g}$ em $1000 \mathrm{ml}$ de agua destilada), conforme recomendado pelo fabricante.

\section{Nested-PCR}

Amostras do sangue venoso foram congeladas em alíquotas de $300 \mu \mathrm{l}$ em microtubos após a colheita e mantidos a $-20^{\circ} \mathrm{C}$ até seu uso. Para a extração do DNA genômico, foi usado o QIAamp ${ }^{\mathrm{TM}}$ DNA blood Mini Kit (QIAGEN ${ }^{\mathrm{TM}}$, EUA), de acordo com as instruções do fabricante. O DNA extraído foi identificado e armazenado a $-20^{\circ} \mathrm{C}$ até seu uso.

A sequência de oligonucleotídios iniciadores ECC 5'-AGAACGAACGCTGGCGGCAAGC 3'e reverso ECB 5'-CGTATTACCGCGGCTGCTGGCA-3' foram usados para amplificar parte do gene 16S de Ehrlichia. Posteriormente, o produto foi submetido ao iniciador ECAN5 5'-CAATTATTTATAGCCTCTGGCTATAGGA3' e à sequência reversa $\mathrm{HE} 3$ 5' TATAGGTACCGTCATTATC T TCCCTAT-3' específicos para $\boldsymbol{E}$. canis, conforme desenvolvido por MURPHY et al. (1998) e padronizado por NAKAGHI et al. (2008) e DAGNONE et al. (2009).

Análise estatística dos resultados obtidos

Os resultados da RIFI e da n-PCR foram submetidos a testes de correlação de Pearson (Instat, Versão 5, GraphPad, EUA) e ao teste de Tukey para comparação de médias e regressões para $\mathrm{P}$ até $10 \%$ com $\mathrm{R}^{2}$ ajustado (SAS/STAT, SAS, EUA). Além disso, foi realizada análise de contingência e teste de quiquadrado. Os resultados obtidos na reação de TUNEL foram avaliados quanto à distribuição Gaussiana pelo teste de Shapiro-Wilk. Quando apresentavam distribuição normal, utilizou-se teste t não pareado, sendo os resultados apresentados como média \pm erro padrão. Quando os resultados não se enquadravam na curva de Gauss, foi aplicado o teste Mann Whitney (não paramétrico) e apresentados como mediana e amplitude. Valores de $\mathrm{P}<0,005$ foram considerados significativos. 


\section{RESULTADOS}

Os animais infectados mostraram positividade para $\boldsymbol{E}$. canis por n-PCR a partir da segunda semana pós inoculação (PI) e por imunofluorescência a partir da terceira semana, sendo o PBMC negativo para todas as leituras. O grupo controle apresentou negatividade em todos os testes realizados. A n-PCR mostrou ser mais precoce na detecção dos animais infectados com $\boldsymbol{E}$. canis do que com as demais técnicas empregadas neste estudo.

Técnica de TUNEL e imunohistoquímica para a detecção de moléculas Bcl-2 e Bax

Houve marcação para a fragmentação genômica in situ nos tecidos estudados nos dois grupos, reconhecida pela presença de grumos pardo amarronzados intranucleares. A marcação por TUNEL foi sempre maior nos tecidos dos animais inoculados do que nos controles (Figura 1). Não foi observada marcação histoquímica no rim em nenhum dos animais dos grupos testados, sendo que os órgãos dos animais inoculados que mostraram marcação mais intensa e difusa foram o baço e os linfonodos (Figura 1B, 1D).

\section{Índices apoptóticos}

No baço, o grupo inoculado apresentou maior índice apoptótico (mediana $=7,02$ variando de 1,471 a 15,02) que o grupo controle (mediana $=2,085$, variando de zero a 4,889), com $\mathrm{p}<0,0001$ (Mann Whitney). Nos linfonodos, o grupo inoculado apresentou maior índice apoptótico (mediana $=6,508$, variando de 0,85 a 18,29 ) que o grupo controle (mediana $=1,843$, variando de zero a 3,902 ), $\operatorname{com} \mathrm{P}<0,0001$. No fígado, o grupo inoculado também apresentou maior índice apoptótico (mediana $=2,198$, variando de zero a 6,667 ) que o grupo controle (mediana $=0,9804$, variando de zero a 3,902), com $\mathrm{P}<0,0001$.

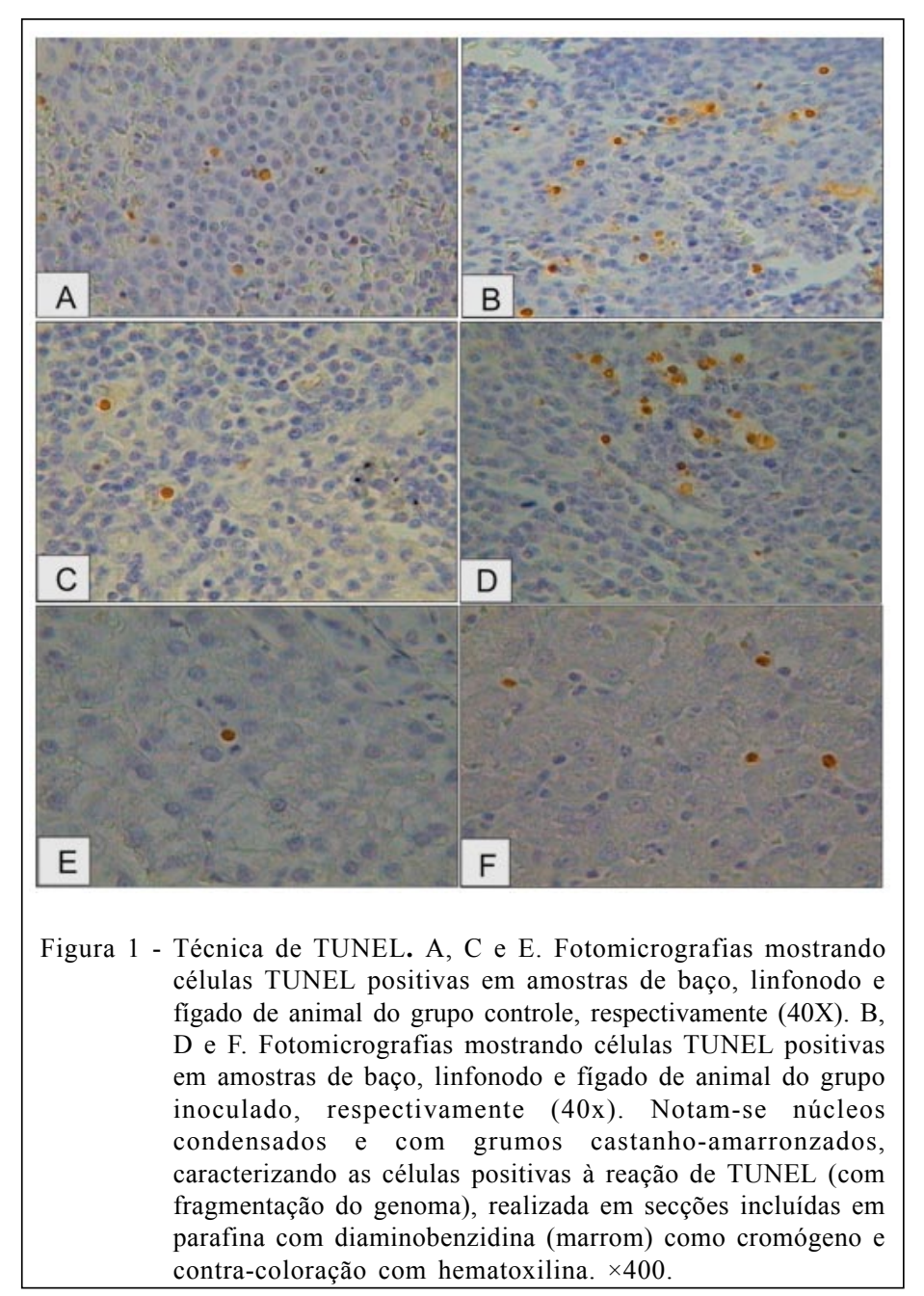

Ciência Rural, v.42, n.8, ago, 2012. 
Técnicas imunohistoquímicas

Nas lâminas tratadas com anticorpos antiBax e anti-Bcl-2, células dos órgãos avaliados exibiram grumos castanho-amarronzados no citoplasma e núcleo (Figura 2), evidenciando marcações positivas para proteínas pró-apoptóticas (Bax) e anti-apoptóticas (Bcl-2) presentes tanto nos animais do grupo inoculado, quanto nos animais do grupo controle. O baço, o linfonodo e o fígado de animais do Grupo Inoculado apresentaram marcação de Bcl-2 mais intensa e mais difusa do que de Bax. No Grupo Controle, verificou-se maior marcação de Bax. No rim também houve maior expressão de Bcl-2 no grupo inoculado. Diferentemente dos outros órgãos, não foi detectada marcação para Bax nas células renais.

\section{DISCUSSÃO}

A escassez de informação específica sobre apoptose na infecção com a $\boldsymbol{E}$. canis e as referências em outras infecções similares (GE et al., 2005; GE \& RIKIHISA, 2006; XIONG et al., 2008) parecem indicar que a $\boldsymbol{E}$. canis também utilize os mecanismos de apoptose em células mononucleares na progressão da infecção. Para se testar essa hipótese, utilizaram-se, neste trabalho, duas técnicas bastante difundidas na avaliação e quantificação da apoptose: TUNEL (GAVRIELI, 1992) e imunohistoquímica para a deteç̧ão das moléculas pró e anti-apoptóticas, Bax (GE \& RIKIHISA, 2006) e Bcl-2 (SPRICK \& WALCZAK, 2004), respectivamente.

Os resultados obtidos neste estudo indicam que, na fase aguda da doença a $\boldsymbol{E}$. canis, ocorre aumento da apoptose nas células de baço, linfonodos e fígado, aumentando-se ao mesmo tempo a imuno-marcação da proteína anti-apoptótica $\mathrm{Bcl} 2$.

A apoptose aqui detectada e quantificada pela técnica de TUNEL ocorre mais intensamente nas células mononucleares. A maior marcação imunohistoquímica de $\mathrm{Bcl}-2$ do que de Bax nos animais do grupo inoculado com $\boldsymbol{E}$. canis observada neste estudo indica que a via intrínseca não é tão importante. A apoptose de células infectadas em nosso estudo pode estar associada à modulação da resposta inflamatória e à resposta imunológica celular do hospedeiro à E. canis. Assim, é provável que a via



Ciência Rural, v.42, n.8, ago, 2012. 
extrínseca seja a mais importante nessas circunstâncias, visto que a estimulação de receptores de morte com seus ligantes (Fas, TNF- $\alpha$ ) permite a ativação de moléculas iniciadoras da apoptose como a caspase 8 (DONEPUDI et al., 2003).

SCHEEL-TOELLNER et al. (2004) demostraram que o A. phagocytophilum, causador da ehrliquiose granulocítica humana, inibe as vias extrínseca e intrínseca na apoptose espontânea de neutrófilos. GE et al. (2005) concluíram que essa infecção inibe a ativação de caspase 3 em neutrófilos humanos. Também foi observado o bloqueio da morte celular programada de neutrófilos humanos induzida por Fas (CD95/Apo-1) na infecção por $\boldsymbol{A}$. phagocytophilum (BORJESSON et al., 2005), sendo sugerido por GE \& RIKIHISA (2006) que Bax é regulada a nível pós-translacional na infecção por este agente. XIONG et al. (2008) demostraram que a infecção com Ehrlichia ewingii atrasa a apoptose espontânea de neutrófilos caninos por estabilização do potencial de membrana mitocondrial. Em outra espécie deste gênero, E. chaffeensis, causadora da ehrliquiose monocítica humana, verifica-se ser esta capaz de inibir $N F-\kappa B$ e apoptose, alterando a expressão de ciclinas e de cinase dependentes de ciclinas e prolongando a sobrevivência do patógeno e da sua célula hospedeira (ZHANG et al., 2004; RIKIHISA, 2006).

A resposta imune de tipo $\mathrm{TH}-1$ com produção de INF- $\gamma$ TNF- $\alpha$ e Il-2 gera imunidade celular, importante na resposta contra as ehrliquias (HARRUS et. al., 1999). Muitos dos patógenos intracelulares podem estimular ou inibir apoptose (GRASSMÉ et al., 2001). Nesse contexto, é importante reconhecer que as vias da sinalização da apoptose são classificadas em via extrínseca (receptores de morte) e via intrínseca (mitocondrial), ativando a cascata de caspases e induzindo a morte celular (SPRICK \& WALCZAK, 2004). Os resultados de imunohistoquímica para Bcl-2 e Bax aqui apresentados indicam que a via intrínseca de apoptose não é importante nos órgãos analisados.

Além disso, os resultados obtidos com base na inoculação in vivo por $\boldsymbol{E}$. canis e enfocando a ocorrência de apoptose e a imunomarcação de moléculas pró e anti apoptose em órgãos como linfonodo, baço, fígado e rim, quando revistos concomitantemente com a literatura científica disponível, apontam para uma situação em que o agente infeccioso tem duas opções: necrosar as células do hospedeiro e obter mais prontamente os nutrientes que precisa para propagar, enfrentando a seguir a reação do hospedeiro, ou irritar menos as células do hospedeiro, causando apoptose e não necrose, de maneira a obter os nutrientes mais lentamente, com isso minimizando as reações do hospedeiro e evadindose da resposta inflamatória. Assim, a inflamação, se adequadamente ativada, eliminaria ou, no mínimo, dificultaria a colonização por essa bactéria, confirmando que o agente etiológico mais bem sucedido é aquele que, apesar de patogênico, consegue o que necessita para sua sobrevivência num ambiente de tolerância, sem induzir uma reação no hospedeiro que prejudicaria sua persistência.

\section{AGRADECIMENTOS}

À Fundação de Amparo a Pesquisa do Estado de Minas Gerais (FAPEMIG) e ao Conselho Nacional de Desenvolvimento Cientifico e Tecnológico (CNPq). À Coordenação de Aperfeiçoamento de Pessoal de Nível Superior (CAPES).

\section{COMITÊ DE ÉTICA E BIOSSEGURANÇA}

O presente trabalho está de acordo com os princípios éticos de experimentação animal e biossegurança (Parecer CETEA/UFMG no 216/09, 10 de março de 2010).

\section{REFERENCIAS}

AGUIAR, D. et al. Diagnóstico sorológico de erliquiose canina com antígeno brasileiro de Ehrlichia canis. Ciência Rural, v.37, p.796-802, 2007. Disponível em: <http://dx.doi.org/ 10.1590/S0103-84782007000300030>. Acesso em: 26 mar. 2012. doi: $10.1590 / \mathrm{S} 0103-84782007000300030$.

BORJESSON, D. et al. Insights into pathogen immune evasion mechanisms: Anaplasma phagocytophilum fails to induce an apoptosis differentiation program in human neutrophils. Journal of Immunology, v.174, p.6364-6372, 2005. Disponível em: <http://www.jimmunol.org/content/174/10/ 6364.long $>$. Acesso em: 26 mar. 2012.

DAGNONE, A. et al. Molecular diagnosis of Anaplasmataceae organisms in dogs with clinical and microscopical signs of ehrlichiosis. Revista Brasileira de Parasitologia Veterinaria, v.18, p.20-25, 2009. Disponível em: <http:// www.scielo.br/scielo.php? script $=$ sci arttext\&pid $=$ S 1984 $29612009000400004 \& \operatorname{lng}=\mathrm{en} \& \mathrm{nrm}=\mathrm{iso} \& \operatorname{tn} \mathrm{ln}=\mathrm{en}>$. Acesso em: 26 mar. 2012. doi: 10.4322/rbpv.01804004.

DELEO, F. Modulation of phagocyte apoptosis by bacterial pathogens. Apoptosis, v.9, p.399-413, 2004. Disponível em: <http:// www.springerlink.com/content/p95g77r5576gw854/>. Acesso em: 26 mar. 2012. doi: 10.1023/B:APPT.0000031448.64969.fa.

DONEPUDI, M. et al. Insights into the regulatory mechanisms for caspase-8 activation. Mollecular Cell, v.11, p.543-549, 2003. Disponível em: <http://www.sciencedirect.com/science/ article/pii/S1097276503000595>. Acesso em: 26 mar. 2012. doi:10.1016/S1097-2765(03)00059-5.

DUMLER, J. et al. Reorganization of genera in the families Rickettsiaceae and Anaplasmataceae in the order Rickettsiales: unification of some species of Ehrlichia with Anaplasma, Cowdria with Ehrlichia and Ehrlichia with Neorickettsia, 
descriptions of six new species combinations and designations of Ehrlichia equi and HGE agent as subjectives synonyms of Ehrlichia phagocytophila. International Journal of Systematic and Evolutionary Microbiology, v.51, p.21452165, 2001. Disponível em: <http://ijs.sgmjournals.org/ content/51/6/2145.full.pdf + html $>$. Acesso em: 23 mar. 2012

GAVRIELI, Y. et al. Identification of programmed cell death in situ via specific labeling of nuclear DNA fragmentation. Journal of Cell Biology, v.119, p.493-501, 1992. Disponível em: <http://www.ncbi.nlm.nih.gov/pmc/articles/PMC2289665/ pdf/jc1193493.pdf $>$. Acesso em: 26 mar. 2012. doi: 00219525/92/11/493/9.

GE, Y. et al. Anaplasma phagocytophilum inhibits human neutrophil apoptosis via upregulation of bfl-1, maintenance of mitochondrial membrane potential and prevention of caspase 3 activation. Cellular Microbiology, v.7, p.29-38, 2005. Disponível em: <http://onlinelibrary.wiley.com/doi/10.1111/ j.1462-5822.2004.00427.x/pdf>. Acesso em: 26 mar. 2012 doi: $10.1111 / \mathrm{j} .1462-5822.2004 .00427$.

GE, Y.; RIKIHISA, Y. Anaplasma phagocytophilum delays spontaneous human neutrophil apoptosis by modulation of multiple apoptotic pathways. Cellular Microbiology. v.8, p.1406-1416, 2006. <http://onlinelibrary.wiley.com/doi/ 10.1111/j.1462-5822.2006.00720.x/pdf>. Acesso em: 26 mar. 2012. doi: $10.1111 / \mathrm{j} .1462-5822.2006 .00720$.x.

GRASSMÉ, H. et al. Molecular mechanisms of bacteria induced apoptosis. Apoptosis. v.6, p.441-445, 2001. Disponível em: $<$ http://www.springerlink.com/content/q63g1n87223v3114/> Acesso em: 26 mar. 2012. doi: 10.1023/A:1012485506972.

HARRUS, S. et al. Recent advances in determining the pathogenesis of canine monocytic ehrlichiosis. Journal of Clinical Microbiology, v.37, p.2745-2749, 1999. Disponível em: <http://www.ncbi.nlm.nih.gov/pmc/articles/PMC85367/ pdf/jm002745.pdf>. Acesso em: 26 mar. 2012. doi: 0095 $1137 / 99 / \$ 04.00+0$.

HESS, P. et al. Experimental Ehrlichia canis infection in the dog does not cause immunosuppression. Veterinary Immunology and Immunopathology, v.109, p.117-125, 2006. Disponivel em: $<\mathrm{http}: / / \mathrm{www}$. sciencedirect.com/science/ article/pii/S0165242705002539>. Acesso em: 26 mar. 2012 doi: $10.1016 /$ j.vetimm.2005.07.027

LABRUNA, M.; PEREIRA, M. Carrapatos em cães no Brasil. Clinica Veterinária, v.30, p.24-32, 2001. Disponível em: $<\mathrm{http}$ ://www.revistaclinicaveterinaria.com.br/v $1 /$ edicoes_ver.php?ver=30> Acesso em 26 mar. 2012.

MANNA, L. et al. First molecular characterization of a granulocytic Ehrlichia strain isolated from a dog in South Italy. Veterinary Journal, v.167, p.224-227, 2004. Disponível em: <http://www.sciencedirect.com/science/article/pii/ S1090023304000516>. Acesso em: 26 mar. 2012. doi:10.1016/ j.tvj1.2004.02.008.

MELO, A. et al. Seroprevalence and risk factors to Ehrlichia spp. and Rickettsia spp. in dogs from the Pantanal Region of Mato Grosso State, Brazil. Ticks Tick-borne Disease. 2011 Disponível em: <http://www.sciencedirect.com/science/article/ pii/S1877959X11000689>. Acesso em: 26 mar. 2012. doi:10.1016/j.ttbdis.2011.09.007.

MORO, L. et al. Apoptosis in canine distemper. Archives of Virology, v.148, p.153-164, 2003. Disponível em: <http:// www.springerlink.com/content/crhye $9 \mathrm{gx} 2 \mathrm{~g} 1 \mathrm{kkpdl} />$. Acesso em: 26 mar. 2012. doi: 10.1007/s00705-002-0903-6.

MURPHY, G. et al. A molecular and serologic survey of Ehrlichia canis, Ehrlichia chaffeensis, and Ehrlichia ewingii in dogs and ticks from Oklahoma. Veterinary Parasitology, v.79, p.325339, 1998. Disponível em: <http://www.sciencedirect.com/ science/article/pii/S0304401798001794>. Acesso em: 26 mar. 2012. doi:10.1016/S0304-4017(98)00179-4.

NAKAGHI, A. et al. Canine ehrlichiosis: clinical, hematological, serological and molecular aspects. Ciência Rural, v.38, p.766-770, 2008. Disponível em: $<$ http://www.scielo.br/scielo.php?pid=S0103$84782008000300027 \&$ script $=$ sci arttext $>$. Acesso em: 26 mar 2012. doi: $10.1590 / \mathrm{S} 0103-84 \overline{7} 82008000300027$

RIKIHISA, Y. Ehrlichia Subversion of host innate responses. Current Opinion in Micro biology, v.9, p.95-101, 2006. Disponível em: <http://www.sciencedirect.com/science/article/ pii/ S1369527405001979>. Acesso em: 26 mar. 2012. doi:10.1016/j.mib.2005.12.003.

RODRIGUES, L. et al. Caspase -3/caspase-8, bax and bcl2 in pulps of human primary teeth with physiological root resorption. International Journal of Paediatric Dentistry, v.22, p.52-59, 2011. Disponível em: < http://www.ncbi.nlm.nih.gov/pubmed?term $=$ Caspase $\% 20$ $3 \% 2 \mathrm{~F}$ c a s a se $-8 \% 2 \mathrm{C} \% 20 \mathrm{~b}$ ax $\% 20$ and $\% 20 \mathrm{bc} 12 \% 2$ 0in\%20pulps\%20of\%20human\%20primary\%20teeth\%20with\% 20 physiological\%20root\%20resorption>. Acesso em: 22 de mar. 2012. doi 10.1111/j.1365-263X.2011.01157.x.Epub 2011 Aug 8.

SCHEEL-TOELLNER, D. et al. Reactive oxygen species limit neutrophil life span by activating death receptor signaling. Blood, v.104, p.2557-2564, 2004. Disponível em: <http:// bloodjournal.hematologylibrary.org/content/104/8 2557.full.pdf + html $>$. Acesso em: 26 mar. 2012. doi:10.1182/ blood-2004-01-0191.

SIARKOU, V. et al. Sequence and phylogenetic analysis of the $16 \mathrm{~S}$ rRNA gene of Ehrlichia canis strains in dogs with clinical monocytic ehrlichiosis. Veterinary Microbiology. v.125, p.304-312, 2007. Disponível em: <http://www.sciencedirect.com/science/article/pii/ S0378113507002684>. Acesso em: 26 mar. 2012. doi:10.1016/ j.vetmic.2007.05.021.

SPRICK, M.; WALCZAK, H. The interplay between the Bcl2 family and death receptor-mediated apoptosis. Biochimica et Biophysica Acta, v.1644, p.125-132, 2004. Disponível em: <http://www.sciencedirect.com/science/article/pii/ S0167488903001794>. Acesso em 26 mar. 2012. doi:10.1016/ j.bbamcr.2003.11.002.

XIONG, Q. et al. Ehrlichia ewingii infection delays spontaneous neutrophil apoptosis throughstabilization of mitochondria Journal of Infectious Diseases, v.197, p.1110-1118, 2008. Disponível em: < http://jid.oxfordjournals.org/content/197/8/ 1110.long>. Acesso em: 26 mar. 2012. doi: 10.1086/533457.

YU XUE-JIE, et al. Restriction and expansion of Ehrlichia strain diversity. Veterinary Parasitology, v.143, p.337-346, 2007. Disponível em: <http://www.sciencedirect.com/science/ article/pii/S0304401706004900>. Acesso em: 26 mar. 2012. doi: $10.1016 /$ j.vetpar.2006.08.027.

ZHANG, J. et al. Survival strategy of obligatory intracellular Ehrlichia chaffeensis: novel modulation of immune response and host cell cycles. Infection and Immunity, v.72, p.498507, 2004. Disponível em: < http://iai.asm.org/content/72/1/ 498.full>. Acesso em: 26 mar. 2012. doi: 10.1128/IAI.72.1.498507.2004 